\title{
Survey of Energy Efficient Tracking and Localization Techniques in Buildings Using Optical and Wireless Communication Media
}

\author{
Tom M. Bruintjes, André B.J. Kokkeler, Georgios Karagiannis and \\ Gerard J.M. Smit \\ University of Twente, Hallenweg 19, 7522 NH Enschede, The Netherlands, \\ t.m.bruintjes@utwente.nl, \\ WWW home page: http://caes.ewi.utwente.nl/
}

\begin{abstract}
This paper presents a survey of beamforming, beamsteering and mobile tracking techniques. The survey was made in the context of the SOWICI project. The aim of this project is to reduce power consumption of data exchanging devices within houses. An optical fiber network is used for data transport to and from rooms whereas wireless transceivers communicate with appliances within the rooms. Using this approach, the aim is to reduce power consumption and exposure to electromagnetic radiation. To realize this, beamforming will be used to only radiate energy in, and receive signals from, the direction of interest. Because appliances within households can move, some of them even relatively fast, the pointing direction of the beam should be steerable. The pointing direction can be deduced from the communication link (beamsteering) or via separate mobile tracking techniques.
\end{abstract}

Keywords: beamforming, beamsteering, mobile tracking

\section{Introduction}

Today's society is confronted with immense challenges such as climate change, depletion of resources and aging society. This has triggered a change in mindset from continuous growth to sustainability (e.g., smart buildings and smart mobility). Today's homes and buildings are responsible for $41 \%$ of the energy consumption in the European Union. They contain many functionalities like domestic appliances, heating, air-conditioning, lighting, electronics and wireless systems that are not responding adequately to actual situations; they do not adapt to actual energy needs and mostly they are not aware of each other due to a lack of overall coordination and control. This leads to unnecessary energy consumption.

Especially in homes and buildings, energy can be saved by using modern technology with situational-aware devices that are able to communicate. Home automation encompasses the increased automation of appliances in residential dwellings through electronic means, to meet the specific needs of the inhabitants. The term "home automation" is used in contrast to the more mainstream 
"building automation", which refers to similar technology for general needs, particularly the automatic or semi-automatic control of lighting, doors and windows, heating, ventilation, air conditioning, etc.. Both home and building automation heavily rely on sensors and actuators that interchange information with control units via reliable communication links. For efficiency reasons, the communication for home/building automation should preferably be integrated with the communication system used for data exchange and multi-media entertainment, which is not the case today. Needless to say that the integrated system should be reliable, flexible, scalable and of course it should consume minimal power. It should support fixed and mobile devices as well as low and high bit-rate devices efficiently. Finally, the wireless communication should work reliably with a minimum of electro-magnetic (EM) emission for energy-efficiency and to eliminate potential health hazards due to exposure to EM radiation in a world full of wireless devices.

By using $60 \mathrm{GHz}$ radio transmission in room-sized pico-cells in combination with adaptive radio pencil beams which are steered to pin-point the (mobile) wireless devices, considerable savings in the energy consumption of the network itself can be achieved. The main goal of this paper is to provide an overview of energy efficient localization and tracking techniques that can be applied in buildings when using optical and wireless communication media. In particular, in this paper, we first introduce the SOWICI project [1] which aims at using energy efficient tracking techniques based on these communication media. Second, as part of this project, existing solutions are surveyed in the areas of Beamforming section 3), Beamsteering section 4) and Mobile Tracking section 5 with an emphasis on the latter. At the end of this paper, we will draw conclusions, relevant to the SOWICI project, based on this survey.

\section{The SOWICI Project}

Within the SOWICI project, which started in 2011, a novel hybrid optical/wireless network architecture is proposed that integrates the home automation network with the high bit-rate in-home network for data transfer and entertainment in a very energy-efficient way. In particular, this integrated network features an optical fiber network backbone between a central control unit and all rooms with wireless transmission inside the rooms. SOWICI supports $60 \mathrm{GHz}$ radio transmission in combination with beamforming to save energy consumption. In addition, the exposure of humans to EM radiation is reduced to a bare minimum. We expect to reduce the radio emission by at least three orders of magnitude.

The general infrastructure, researched within the SOWICI project is depicted in Fig. 1. Wireless transmitters (Radio Access Points, RAPs) are placed in a $(4 \times 4)$ rectangular planar array configuration such that beamforming becomes possible by means of constructive interference. Pointing the beams to the Mobile Devices (MDs) present in a room will yield considerable savings in the energy consumption of the network. However, MDs can move around in the building. Beamforming therefore needs to be adaptive. 


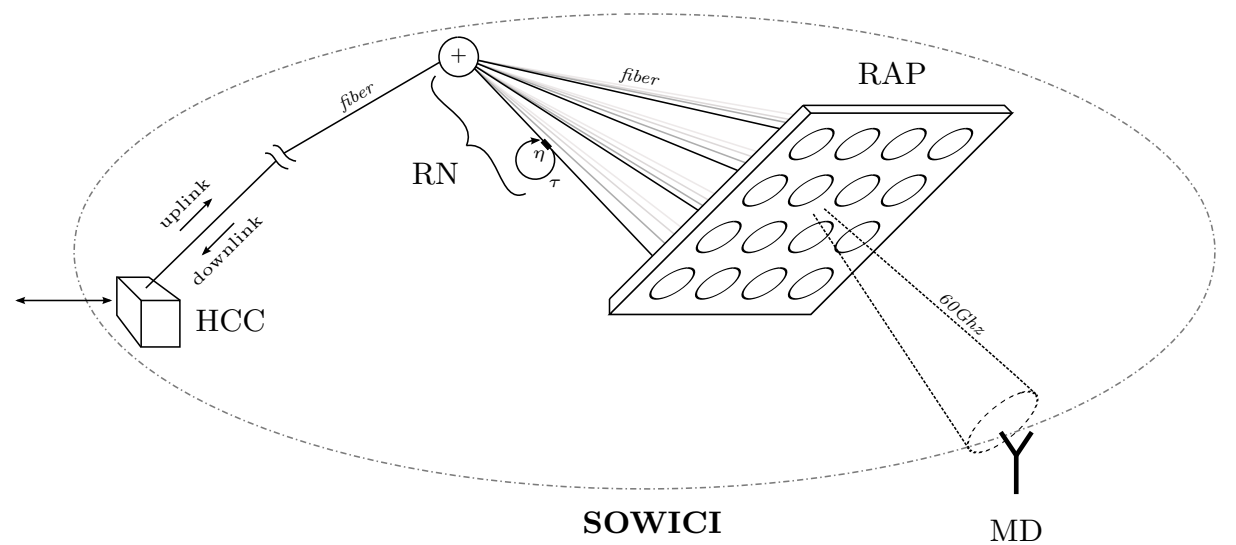

Fig. 1. SOWICI Infrastructure

To steer and/or shape a beam, a variable delay $\tau$ and gain $\eta$ need to be applied to each of the antenna elements of the RAP. To drive the antenna elements with the appropriate delay and gain, an integrated optical circuit is used. Although this Reconfigurable Node (RN) is responsible for physically inducing the delays needed for constructive interference, the control intelligence for localization and tracking is located in the centralized Home Communication Center (HCC). The fiber that connects the HCC to the different RAPs therefore conveys both the actual data for the MDs and the RN configuration information to control the angle and shape of the beam remotely from the HCC. To check the appropriateness of the proposed infrastructure, a survey of existing alternatives in beamforming and tracking mobiles in general was made.

\section{Beamforming}

Radio beams can be steered by using beamforming techniques, often also referred to as phased array techniques. Basic theory on this subject can be found in [56]. Until recently, mainly narrowband beamforming has been considered because of ease of implementation. The term 'narrowband' is used for signals whose bandwidth is much smaller than their center frequency (generally $1 \%$ or less). In other cases, a signal is 'wideband'. Wideband beamforming techniques are studied in detail more and more 32. In phased array antennas, differences in the phases (narrowband beamforming) or time (wideband beamforming), determine the shape and direction of the beam. For this, accurate and tuneable shifting of phases or time delays need to be accomplished, which is particularly challenging when microwave radio signals with complex modulation formats are involved and fast beamsteering is required.

Traditionally, beamforming is realized in electronic circuits in contrast with optical beamforming discussed later on. A time delay or phase shift can be realized at different stages [57]. 
- RF beamforming realizes beamforming at RF frequencies and is done fully analogue [44.

- LO Beamforming performs the beamforming operation at intermediate frequencies (IF) where phase shifts are introduced in the local oscillator (LO) signals. This type of beamforming is inherently narrowband.

- IF beamforming performs beamforming at intermediate frequencies (IF) and generally in the digital domain. This has the advantage that the flexibility and computational capacity of digital processors can be exploited to the full.

- Beamforming by optical techniques was achieved by deploying bulky dispersive fiber delay lines, and dispersive micro-ring structures 36 33. Only 1-dimensional beamsteering was addressed.

There are several application areas for beamforming.

- One of the first areas to adopt these techniques was radar. Phased arrays for radar have been used since the 1950s to detect, locate and follow reflecting objects or targets [58] .

- Phased arrays are also used to construct images of celestial object within radio astronomy. A Radio Telescope for astronomy, exploiting phased array technology that is currently operational is LOFAR [58].

- Within telecommunications, beamforming is used within GSM and UMTS networks mainly in the downlink (from base station to mobile) [21] 20, 41, within WLAN 62 and LTE 8 . During the last few years, multi-antenna techniques at both the transmitter and receiver have also been introduced in the latest wireless standards (e.g., IEEE803.11n [7]). However, these techniques are applied in a highly scattering environment where signals arrive from different directions. In this case, the techniques used are generally referred to as MIMO [19].

A significant challenge associated with beamforming is how to form the beam in such a way that the phased array antenna can transmit (almost) simultaneously different content towards multiple mobile devices located in a room. Two alternatives can be distinguished:

- For each mobile device located in a room a time slot is allocated. During a time slot a beam is formed in such a way that the phased array antenna can transmit only to one mobile device. The disadvantages of this solution are (1) the switching in time; (2) the reconfiguration of the phased array antenna elements; (3) transmission of the information needs to be accomplished within the given time slot.

- More than one beams are formed simultaneously and sent to the different mobile devices in the room. The main disadvantage of this option is that each beam is formed using a smaller in size phased array (i.e., only a subset of the phased array antenna elements are used).

Furthermore, we intend to stay IEEE 802.15.3C [18] compliant within SOWICI. The IEEE 802.15.3C standard defines a central controlled network topology and 
a TDMA based MAC protocol for Wireless Personal Area Networks (WPANs) operating in the unlicensed $60 \mathrm{GHz}$ band. It also specifies an optional codebook beamforming protocol, which is based on scanning discrete sectors section 5.2.

\section{Beamsteering}

For stationary devices their location can be detected at the start of a communication session during a training sequence [47. However, for moving mobile devices the position needs to be tracked continuously. Algorithms that adapt steering vector weights $(\tau$ and $\eta$ ) to steer the beam are called adaptive beamforming algorithms. These type of algorithms can be classified into three subclasses [2]: Temporal reference beamforming algorithms, Spatial reference beamforming algorithms and Blind beamforming algorithms.

- In Temporal reference beamforming, known temporal signal properties are used to adjust the beamsteering 61 .

- Spatial reference beamforming uses Direction-of-Arrival (DoA) algorithms to estimate the direction of the impinging signal. Well-known examples of DoA algorithms are MUSIC [47 and ESPRIT [46. Most spatial reference algorithms are inappropriate for real-time beamsteering because of their high computational costs.

- Blind beamforming algorithms use statistical properties of the desired signal to determine the angle of arrival of the signal. An example of a blind beamforming algorithm is the Constant Modulus Algorithm (CMA) [49] [50]. Blind beamforming algorithms are efficient since they do not need a-priori information about channel characteristics. Thus, the initial training phase of an adaptive equalizer is avoided, eliminating potential losses in channel capacity. An extended version of CMA can be used to adapt the steering vector weights to compensate for movement of mobiles [49] 50, but such an algorithm is quite computationally intensive.

\section{$5 \quad$ Mobile Localization and Tracking}

Instead of using one of the beamsteering algorithms mentioned above, a separate mobile tracking technique can be used to obtain the information about the desired pointing direction of the beamformer. In this section, a broad survey of mobile tracking techniques is presented. The purpose is to become familiar with mobile tracking in a broad sense and to assess the applicability of (parts of) the techniques to be used within the SOWICI project.

Currently mobile users can roam ubiquitously and have access from many locations. Therefore, the knowledge of the physical locations of mobile user devices, such as phones, laptops, is important in network operation \& planning and in several applications such as location based services and low enforcement services. The procedure required for the estimation of such physical locations (e.g., longitude, latitude, altitude) is usually denoted as localization, which is 
estimated relative to a reference position. The wireless device whose location is to be estimated is usually called Localization Node (LN) and the network entity with known location is usually called Localization Base Station (LBS). One important performance measure of localization schemes is the achieved accuracy on estimating the exact physical position of devices. Several surveys and classification on localization solutions have been published, such as 38, 35. 10 .

\subsection{Classification of Localization schemes}

Localization is classified based on (a) area of deployment, (b) used wireless technology physical layer, (c) measured localization physical parameter, (d) location lookup table, (e) the estimation technique, (f) the localizing entity and (g) the supported security.

Area of deployment Localization schemes depend on the area of deployment, due to the differences in network topology, number of users and available resources for such networks. This category can be subdivided into Wide Area Localization (WAL), Local Area Localization (LAL) and Ad-Hoc Localization (AHL). The WAL category is characterized by predominantly outdoor deployments, such as:

- Global Navigation Satellite System (GNSS) Galileo [14 and Global Positioning System (GPS) [22, Differential GPS [25, Assisted GPS [17.

- Cellular network based localization solutions [55].

The LAL category is characterized by predominantly indoor deployments, such as:

- Active Batch [13].

- Wireless LAN localization solutions 39.

The AHL category is characterized by the use of ad-hoc networks and wireless sensor networks which typically are power constrained and may be heterogeneous in nature. Examples of localization solutions that are based on wireless sensor networks are described in [35], which can be:

- Centralized based, where centralized information already exists such as road traffic monitoring, health monitoring and where the measurement data of all nodes are collected in a central processing unit 29].

- Distributed based, where each node relies on self-localization using the measured distances and the local information from its neighbouring nodes 28].

Example solutions that are based on mobile ad-hoc networks are e.g., [1].

Used Wireless Technology The used wireless technology and in particular its physical layer, influences significantly the localization solution. This category can be subdivided into:

- Infrared (IR) solutions [59].

- Ultrasound (US), where the signals can propagate through walls [43.

- Radio Frequency (RF), which is the most commonly used type, e.g., GPS based systems [22] [17]. 
Measured Localization Physical Parameter The measured localization physical parameter of the used radio link between the LN and multiple LBSs is used to estimate the location of LN. Several physical parameter values can be used to estimate the location of the LN. The most common are:

- Signal Strength (SS) or Received Signal Strength Indicator (RSSI) [5].

- Link Quality Indicator (LQI), which is a metric of the current quality of the received signal. Not purely based on RSSI but often a combination of RSSI and Signal to Noise Ratio (SNR) [53].

- Time of Arrival (ToA), which is the time taken by wireless packets (or signals) to travel from a transmitter to a receiver at a certain distance 37. Differential Time of Arrival (DTOA) is used to avoid complex time synchronization 60. Two signals traveling at different propagation speeds are sent out and then quantify the difference in time of arrival. If both signal propagation speeds are known, a distance can be derived from the difference measurement.

- Connectivity (Cnn), which indicates that the $\mathrm{LN}$ is in the transmission range of an LBS [59].

- Fingerprinting uses a signature (or a fingerprint), such as radio link, optical, acoustic, or motion attributes, to identify a certain logical location [4].

- The techniques discussed in section 4 can be considered as techniques that measure physical parameters as well.

Location Lookup Table (LLT) The location lookup table (LLT) is used to map the physical parameter values onto the various locations within the deployment site and prior to the actual localization process. Different types of LLTs are known. The most common ones are:

- Measurement based, where the physical parameter at various locations on the deployment site is measured in order to construct the LLT [3].

- Agent based, where the LLTs is built using readings from agents that are placed at various locations to perform certain automatic measurements without requiring extensive manual costs [31.

- Model based, where analytical/empirical models are used to estimate the values of the physical parameter at any location within the deployment site [5].

Estimation Technique An estimation technique can be used to calculate the position of the LN based on the available LLT and the current measurements of the physical parameter within the deployment site. The current location of the LN is estimated to be the physical location that has physical parameter values closest to the current measured values. Two types of estimation techniques are used:

- Deterministic estimation, where statistical parameters such as measured physical parameter mean or median between LN and multiple LBSs are used for a robust multilateral estimation [12]. 
- Probabilistic estimation, where the position of LNs can be estimated based on the statistical distribution of the physical parameter at different locations within the deployment site [13.

Localizing Entity The described estimation techniques can be carried out by either the LN or the LBS. Further subdivision is possible by distinguishing between:

- Client based localization, where the LN determines its position by monitoring the signals and/or messages received from the LBSs 13 .

- Network based localization, where the network determines the position of a LN by measuring the physical parameter values of the signals and/or messages sent by the LN towards several LSBs [5].

Supported Security The security category is characterized by whether a localization technique is resistant to location spoofing and to security attacks. This category is subdivided in:

- Open, where security is not supported meaning that devices could easily spoof their position to various locations [59].

- Secure, where location spoofing and security attacks on a localization scheme are protected [3].

\subsection{Indoor Localization Techniques}

Most applicable to SOWICI are indoor localization techniques. Such mechanisms have been studied extensively in [23] and [35]. Focusing on wireless technology, the following can be distinguished:

- GPS-based using an indoor GPS technique [6].

- RFID-based using RSS information to calculate the location of RFID tags 9.

- Cellular-based using the mobile cellular network to estimate indoor positioning, when considering that the building is covered by several LBSs [54].

- Ultra Wide Band(UWB)-based using ultra-short pulses with a low duty cycle permitting an accurate determination of the Time of Arrival [34.

- WLAN-based using IEEE 802.11 for positioning by e.g., adding a location server [5].

- Bluetooth-based using e.g., RSSI values for locating specific Bluetooth tags [52].

- IEEE 802.15.4-based 26]. Several tracking techniques are developed to measure the distance between two or more IEEE 802.15.4 compliant sensor devices. Such techniques are based on (1) combination of signal time-of-flight and phase measurements [48, (2) RSSI measurements [45] 45, 15] (3) Time (Difference) of Arrival [42] [51, (4) using Ultra Wide Band [40, (5) using Angle of Arrival [40], (6) using Radio Interferometric Positioning (RIP) 24] [16]. 
Localization using phased arrays is possible by:

- Direction or Angle of Arrival (AoA) algorithms such as MUSIC [47 and ESPRIT [46]. In order to derive the direction of arrival these algorithms consider that the received signals are narrowband and that phase differences between the individual elements or the array are measurable.

- The extended Constant Modulus Algorithm (CMA), see section 4 can be used for beamsteering and mobile tracking, which is able to adapt the steering vector weights to compensate for movement of the LNs 49, 50, 30. This algorithm also considers that the received signals are narrowband and that phase information is available.

- Systematically scanning an area by changing the beam direction, using an antenna array, and by measuring the signal strength [33. The LN is located in the direction where the signal strength is the highest. Such scanning techniques require a search algorithm to be able to localize LNs that are fast moving but do not require that the received signals are narrowband. An example of such an algorithm can be found in the the IEEE 802.15.3C standard 27. A beamforming protocol (codebook beamforming) is proposed, which is based on scanning sectors with increasing granularity. The protocol consists of three stages: sector-level (coarse) training, beam-level (fine) training, and an optional tracking phase. The division of the stages facilitates a significant reduction in setup time compared with beamforming mechanisms using exhaustive searching. Moreover, the protocol employs only discrete phase shifts, which simplifies the problem compared to continuously tuneable beamforming. Scanning algorithms often assume a discrete beamformer (i.e., the best beam is selected from a limited set of beam patterns).

- Applying small perturbations to the current steering weights and evaluating if and in which direction the RSS/RSSI is better [18. Perturbation techniques are closely related to scanning but are usually applicable to continuous beamformers.

\subsection{Localization Selection Criteria}

In order to select a localization scheme several criteria can be applied:

- Cost of deployment, which constitutes the initial deployment costs along with maintenance costs.

- Required accuracy, which is influenced mainly by the choice of the applied wireless technology physical layer, measured localization physical parameter and the estimation technique.

- Resource requirements and computational complexity, which measures the computation complexity and the resources, such as radio resources, required by a certain localization scheme.

- Effects on underlying network, which measures the impact of a localization scheme on the used underlying communication network. 
- Security and privacy, which measures whether a localization scheme is resistant to e.g., intruders that try to type of environment, gain access and disrupt the operation of a system and whether a localization scheme protects the identity of users while they are being localized.

- Type of environment, which affects the choice of the wireless technology physical layer, localization lookup table and the estimation technique. Some significant characteristics that need to be taken into account when considering the use of a multi-array receiver antenna for localization are related to whether the localization algorithm can receive/measure the phase information of the received signals and whether the received signals are narrowband or wideband.

- Effects on energy consumption, which measures the impact of a localization scheme on the consumed energy

\subsection{Identifying the Appropriate Techniques for SOWICI}

From the selection of criteria mentioned in the previous section, energy consumption is the most important for SOWICI. The resource requirements and computational complexity of the technique used are closely related to energy consumption. Hence, these are our primary concerns at this early stage in the research. Ideally we would like SOWICI to localize MDs using only the phased array. The other techniques that have been discussed need additional equipment which is likely to increase the costs of deployment. In addition we think that a mixture of techniques will result in a less elegant solution. However, if complementary techniques add up to the energy consumption or help reduce it remains to be answered. Because we intend to bring energy consumption to a bare minimum, other solution have to be considered.

Table 1. Selection Criteria applied to Indoor Localization Techniques

\begin{tabular}{|c|c|c|c|c|c|}
\hline & Cost & Accuracy & Resources & Environment & Energy \\
\hline GPS & $\checkmark x$ & $\checkmark x$ & $\checkmark x$ & $x x$ & $x x$ \\
\hline RFID & $\checkmark$ & $\checkmark$ & $\sqrt{ } x$ & $\checkmark \checkmark$ & $\checkmark \checkmark$ \\
\hline GSM & $\checkmark x$ & $\sqrt{x}$ & $\checkmark x$ & $x x$ & $x x$ \\
\hline WiFi & $\checkmark$ & $\checkmark x$ & $\checkmark$ & $\checkmark \checkmark$ & $\checkmark x$ \\
\hline Bluetooth & $\checkmark \checkmark$ & $\checkmark$ & $\checkmark$ & $\checkmark \checkmark$ & $\checkmark$ \\
\hline IEEE 802.15 .4 & $\checkmark \checkmark$ & $\checkmark \checkmark$ & $\checkmark \checkmark$ & $\checkmark \checkmark$ & $\checkmark \checkmark$ \\
\hline $\begin{array}{l}\text { IEEE 802.15.3C } \\
\text { (beamforming) }\end{array}$ & $\checkmark$ & $\checkmark \checkmark$ & $\checkmark x$ & $\checkmark \checkmark$ & $\checkmark \checkmark$ \\
\hline
\end{tabular}

Table 1 shows the selection criteria from section 5.3 that we think are most important for SOWICI applied to the techniques enumerated insection 5.2. From the techniques that are complementary to the phased array, IEEE 802.15.4 based technology appears to match best with the goals of the SOWICI project. 


\section{Conclusion and Future Work Within SOWICI}

This paper presents a survey of beamforming, beamsteering and mobile tracking techniques. The information presented in this paper has been used within the SOWICI project to identify two main beamsteering and mobile tracking research directions. The first direction is to use the communication signals themselves for directing the beam. In SOWICI, beamforming is realized optically (RF beamforming) and is aimed to comply with the IEEE 802.15.3C standard [18. This means signals are wideband and phase information is lost due to signal summation before analogue to digital conversion. Under these conditions none of the matured Direction-of-Arrival techniques like MUSIC, ESPRIT or CMA can be employed. Within this direction further research will therefore focus on other techniques that are based on RSSI measurements. Such techniques are for example scanning or the approaches based on perturbation, which were discussed in section 5.2. The second direction is to use a separate indoor localization technique to determine the position of the MD and then use this information to point the beam. One of the options that will be considered for further research is localization by means of IEEE 802.15 .4 based technology. The final SOWICI architecture will be assessed based on the selection criteria mentioned in the previous section with an emphasis on energy consumption.

\section{References}

1. SOWICI (2011), http://www.sowici.nl

2. Allen, B., Ghavami, M.: Adaptive Array Systems: Fundamentals and Applications. John Wiley \& Sons Inc (2005)

3. Anjum, F., Pandey, S., Agrawal, P.: Secure localization in sensor networks using transmission range variation. In: IEEE International Conference on Mobile Adhoc and Sensor Systems Conference. pp. 9-pp. IEEE (2005)

4. Azizyan, M., Constandache, I., Roy Choudhury, R.: Surroundsense: mobile phone localization via ambience fingerprinting. In: Proceedings of the 15 th annual international conference on Mobile computing and networking. pp. 261-272. MobiCom '09, ACM, New York, NY, USA (2009), http://doi.acm.org/10.1145/1614320. 1614350

5. Bahl, P., Padmanabhan, V.: Radar: An in-building rf-based user location and tracking system. In: Nineteenth Annual Joint Conference of the IEEE Computer and Communications Societies (INFOCOM 2000). vol. 2, pp. 775-784. IEEE (2000)

6. Barnes, J., Rizos, C., Wang, J., Small, D., Voigt, G., Gambale, N.: High precision indoor and outdoor positioning using locatanet. Journal of Global Positioning System 2(2), 73-82 (2003)

7. Behzad, A.: Radio design for mimo systems with an emphasis on ieee 802.11n. In: Course presented at IEEE International Solid-State Circuits Conference, San Francisco (2007)

8. Boudreau, G., Panicker, J., Guo, N., Chang, R., Wang, N., Vrzic, S.: Interference coordination and cancellation for $4 \mathrm{~g}$ networks. IEEE Communications Magazine 47(4), 74-81 (2009)

9. Bouet, M., Dos Santos, A.: Rfid tags: Positioning principles and localization techniques. In: Wireless Days (WD'08). pp. 1-5. IEEE (2008) 
10. Boukerche, A., Oliveira, H., Nakamura, E., Loureiro, A.: Localization systems for wireless sensor networks. IEEE Wireless Communications 14(6), 6-12 (2007)

11. Boukerche, A., Oliveira, H., Nakamura, E., Loureiro, A.: Vehicular ad hoc networks: A new challenge for localization-based systems. Computer Communications 31(12), 2838-2849 (2008)

12. Čapkun, S., Hamdi, M., Hubaux, J.: Gps-free positioning in mobile ad hoc networks. Cluster Computing 5(2), 157-167 (2002)

13. Castro, P., Chiu, P., Kremenek, T., Muntz, R.: A probabilistic room location service for wireless networked environments. In: Ubicomp 2001: Ubiquitous Computing. pp. 18-34. Springer (2001)

14. Commission, E., et al.: Galileo-mission high level definition. EMRF Doc. 5(5), 2 (2002)

15. Dil, B., Havinga, P.: On the calibration and performance of rss-based localization methods. In: Internet of Things (IOT 2010). pp. 1-8. IEEE Computer Society (November 2010)

16. Dil, B., Havinga, P.: Stochastic radio interferometric positioning in the 2.4ghz range. In: Proceedings of the 9th ACM Conference on Embedded Networked Sensor Systems. pp. 108-120. SenSys'11, ACM, New York, NY, USA (2011)

17. Djuknic, G., Richton, R.: Geolocation and assisted gps. Computer 34(2), 123-125 (2001)

18. Fakharzadeh Jahromi, M.: Optical and Microwave Beamforming for Phased Array Antennas. Ph.D. thesis, University of Waterloo (2010)

19. Gesbert, D., Shafi, M., Shiu, D., Smith, P., Naguib, A.: From theory to practice: an overview of mimo space-time coded wireless systems. IEEE Journal on Selected Areas in Communications 21(3), 281-302 (2003)

20. Godara, L.: Aapplications of antenna arrays to mobile communications ii. beamforming and direction-of-arrival considerations. Proceedings of the IEEE 85(8), 1195-1245 (1997)

21. Godara, L.: Applications of antenna arrays to mobile communications i. performance improvement, feasibility, and system considerations. Proceedings of the IEEE 85(7), 1031-1060 (1997)

22. GPS, N.: User equipment introduction. Department of Defense Document MZ10298 1 (1996)

23. Gu, Y., Lo, A., Niemegeers, I.: A survey of indoor positioning systems for wireless personal networks. IEEE Communications Surveys \& Tutorials 11(1), 13-32 (2009)

24. Havinga, P., et al.: A feasibility study of rip using 2.4ghz 802.15.4 radios. In: IEEE 7th International Conference on Mobile Ad Hoc and Sensor Systems (MASS). pp. 690-696. IEEE (2010)

25. Hofmann-Wellenhof, B., Lichtenegger, H., Collins, J.: Global positioning system. theory and practice. Global Positioning System. Theory and practice. 1 (1993)

26. IEEE: Ieee standard for information technology - telecommunications and information exchange between systems - local and metropolitan area networks specific requirements part 15.4: Wireless medium access control (mac) and physical layer (phy) specifications for low-rate wireless personal area networks (lr-wpans). IEEE Std 802.15.4-2003 pp. 1-670 (2003)

27. IEEE: Wireless medium access control (mac) and physical layer (phy) specifications for high rate wireless personal area networks (wpans) amendment 2: Millimeterwave-based alternative physical layer extension. IEEE Std 802.15.3c-2009 (Amendment to IEEE Std 802.15.3-2003) pp. 1-187 (12 2009) 
28. Ihler, A., Fisher III, J., Moses, R., Willsky, A.: Nonparametric belief propagation for self-localization of sensor networks. IEEE Journal on Selected Areas in Communications 23(4), 809-819 (2005)

29. Kannan, A., Mao, G., Vucetic, B.: Simulated annealing based localization in wireless sensor network. In: The IEEE Conference on Local Computer Networks. pp. 15-22. IEEE (2005)

30. K.C.H., B., van de Burgwal M.D., K.C., R., A.B.J., K., G.J.M., S.: Angular cma: A modified constant modulus algorithm providing steering angle updates. In: The Seventh International Conference on Wireless and Mobile Communications (ICWMC 2011). pp. 42-47. ThinkMind (June 2011)

31. Krishnan, P., Krishnakumar, A., Ju, W., Mallows, C., Gamt, S.: A system for lease: Location estimation assisted by stationary emitters for indoor $\mathrm{rf}$ wireless networks. In: Twenty-third Annual Joint Conference of the IEEE Computer and Communications Societies (INFOCOM 2004). vol. 2, pp. 1001-1011. IEEE (2004)

32. Liu, W., Weiss, S.: Wideband Beamforming: Concepts and Techniques. Wiley (2010)

33. Madsen, C., Lenz, G.: Optical all-pass filters for phase response design with applications for dispersion compensation. IEEE Photonics Technology Letters 10(7), 994-996 (1998)

34. Mahfouz, M., Fathy, A., Kuhn, M., Wang, Y.: Recent trends and advances in uwb positioning. In: IEEE MTT-S International Microwave Workshop on Wireless Sensing, Local Positioning, and RFID (IMWS 2009). pp. 1-4. IEEE (2009)

35. Mao, G., Fidan, B., Anderson, B.: Wireless sensor network localization techniques. Computer Networks 51(10), 2529-2553 (2007)

36. Meijerink, A., Roeloffzen, C., Zhuang, L., Marpaung, D., Heideman, R., Borreman, A., van Etten, W.: Phased array antenna steering using a ring resonator-based optical beam forming network. In: Symposium on Communications and Vehicular Technology. pp. 7-12. IEEE (2006)

37. Nájar, M., Huerta, J., Vidal, J., Castro, J.: Mobile location with bias tracking in non-line-of-sight. In: IEEE International Conference on Acoustics, Speech, and Signal Processing (ICASSP'04). vol. 3, pp. iii-956. IEEE (2004)

38. Pandey, S., Agrawal, P.: A survey on localization techniques for wireless networks. Journal of the Chinese Institute of Engineers 29(7), 1125-1148 (2006)

39. Pandey, S., Kim, B., Anjum, F., Agrawal, F.: Client assisted location data acquisition scheme for secure enterprise wireless networks. In: IEEE Wireless Communications and Networking Conference. vol. 2, pp. 1174-1179. IEEE (2005)

40. Patwari, N.: Location Estimation in Sensor Networks. Ph.D. thesis, University of Michigan (2005)

41. Pedersen, K., Mogensen, P., Ramiro-Moreno, J.: Application and performance of downlink beamforming techniques in umts. IEEE Communications Magazine 41(10), 134-143 (2003)

42. Pichler, M., Schwarzer, S., Stelzer, A., Vossiek, M.: Positioning with moving ieee 802.15.4 (zigbee) transponders. In: IEEE MTT-S International Microwave Workshop on Wireless Sensing, Local Positioning and RFID (IMWS 2009). pp. 1-4. IEEE (2009)

43. Priyantha, N., Chakraborty, A., Balakrishnan, H.: The cricket location-support system. In: Proceedings of the 6th Annual International Conference on Mobile Computing and Networking. pp. 32-43. ACM (2000)

44. Razavi, B.: RF Microelectronics. Prentice Hall (2011) 
45. Robles, J., Tromer, S., Hidalgo, J., Lehnert, R.: A high configurable protocol for indoor localization systems. In: International Conference on Indoor Positioning and Indoor Navigation (IPIN). pp. 1-7. IEEE (2011)

46. Roy, R., Kailath, T.: Esprit-estimation of signal parameters via rotational invariance techniques. IEEE International Conference on Acoustics, Speech, and Signal Processing (ICASSP'89) 37(7), 984-995 (1989)

47. Schmidt, R.: Multiple emitter location and signal parameter estimation. IEEE Transactions on Antennas and Propagation 34(3), 276-280 (1986)

48. Schwarzer, S., Vossiek, M., Pichler, M., Stelzer, A.: Precise distance measurement with ieee 802.15.4 (zigbee) devices. In: IEEE Radio and Wireless Symposium. pp. 779-782. IEEE (2008)

49. Silva, M., Miranda, M.: Tracking issues of some blind equalization algorithms. IEEE Signal Processing Letters 11(9), 760-763 (2004)

50. Silva, M., Nascimento, V.: Tracking analysis of the constant modulus algorithm. In: IEEE International Conference on Acoustics, Speech and Signal Processing (ICASSP 2008). pp. 3561-3564. IEEE (2008)

51. Stelzer, A., Pourvoyeur, K., Fischer, A.: Concept and application of lpm-a novel 3 -d local position measurement system. IEEE Transactions on Microwave Theory Techniques 52, 2664-2669 (december 2004)

52. Subramanian, S., Sommer, J., Zeh, F., Schmitt, S., Rosenstiel, W.: Pbil pdr for scalable bluetooth indoor localization. In: Third International Conference on Next Generation Mobile Applications, Services and Technologies (NGMAST'09). pp. 170-175. IEEE (2009)

53. Tang, L., Wang, K.C., Huang, Y., Gu, F.: Channel characterization and link quality assessment of ieee 802.15.4-compliant radio for factory environments. Industrial Informatics, IEEE Transactions on 3(2), 99 -110 (may 2007)

54. Varshavsky, A., de Lara, E., Hightower, J., LaMarca, A., Otsason, V.: Gsm indoor localization. Pervasive and Mobile Computing 3(6), 698-720 (2007)

55. Venkatraman, S., Caffery Jr, J.: Hybrid toa/aoa techniques for mobile location in non-line-of-sight environments. In: IEEE Wireless Communications and Networking Conference (WCNC 2004). vol. 1, pp. 274-278. IEEE (2004)

56. Visser, H.: Array and Phased Array Antenna Basics. John Wiley \& Sons Inc (2005)

57. van Vliet, F.: Trends in wideband phased-array front-ends. In: European Radar Conference, 2007. (EuRAD 2007). pp. 154-157. IEEE (2007)

58. de Vos, M.: Lofar: the first of a new generation of radio telescopes. In: IEEE International Conference on Acoustics, Speech, and Signal Processing (ICASSP'05). vol. 5, pp. 865-868. IEEE (2005)

59. Want, R., Hopper, A., Falcao, V., Gibbons, J.: The active badge location system. ACM Transactions on Information Systems (TOIS) 10(1), 91-102 (1992)

60. Whitehouse, K., Culler, D.: Calibration as parameter estimation in sensor networks. In: Proceedings of the 1st ACM international workshop on Wireless sensor networks and applications. pp. 59-67. WSNA '02, ACM, New York, NY, USA (2002), http://doi.acm.org/10.1145/570738.570747

61. Winters, J.: Signal acquisition and tracking with adaptive arrays in the digital mobile radio system is-54 with flat fading. IEEE Transactions on Vehicular Technology 42(4), 377-384 (1993)

62. Zetterberg, P., Bengtsson, M., McNamara, D., Karlsson, P., Beach, M.: Performance of multiple-receive multiple-transmit beamforming in wlan-type systems under power or eirp constraints with delayed channel estimates. In: IEEE 55th Vehicular Technology Conference (VTC Spring 2002). vol. 4, pp. 1906-1910. IEEE (2002) 